Pacific Journal of Mathematics

DISTINGUISHING A PLANE CURVE FROM OTHER CURVES 


\title{
DISTINGUISHING A PLANE CURVE FROM OTHER CURVES SIMILAR TO IT
}

\author{
M. S. Keane AND S. J. Sidney
}

\begin{abstract}
If $C$ is a convex or smooth simple closed plane curve, then there is a finite subset $E$ of $C$ such that any plane curve which is similar to $C$ and passes through all the points of $E$ must coincide with $C$. Generalizations to compacta in euclidean spaces are given; on the other hand, there are simple closed plane curves for which no such finite subsets exist. Circles are the only plane continua which separate the plane for which three points suffice, and even for a convex polygon the number of points required may be arbitrarily large.
\end{abstract}

1. Introduction. A circle is determined by (any) three of its points. The aim of this paper is to examine analogous statements for other compact sets in a euclidean space, and especially for more general simple closed plane curves. Precisely, if $K$ is compact, must $K$ contain a finite subset $E$ such that whenever $K^{\prime}$ is similar to $K$ and contains all the points of $E$, it follows that $K^{\prime}=K$ ? In general the reply is negative even for simple closed plane curves (Example 7), but the following result (the original objective of this work) is true.

THEOREM 1. If $C$ is a convex or smooth simple closed plane curve, then $C$ contains a finite subset $E$ such that $C^{\prime} \sim C$ and $E \subset C^{\prime}$ together imply $C^{\prime}=C$.

Here and throughout, $\sim$ denotes similarity and "smooth" means continuously differentiable. In $\S 2$ we prove generalizations and refinements of Theorem 1 in stages, using finite subsets of $C$ to successively force $C$ " to be congruent to $C$, have the same "spanning circle" as $C$, and finally coincide with $C$. It will become clear that, for general $K$, the only possible obstacle to an affirmative reply to our question is the absence of any finite subset (consisting of at least two points) of $K$ of which $K$ contains a smallest copy (Theorem 5). Taking advantage of this perspective, in $\S 3$ a result (Theorem 6) is proved which shows that, for instance, rather general piecewise smooth objects can be added to the list begun in Theorem 1 , and a simple closed plane curve for which no finite subset works is constructed. Section 4 is devoted to estimating how large a finite subset is required for various particular plane sets; in particular (Theorem 8) if a continuum separates the plane, it requires at least four points 
unless it is a circle. In $\S 5$ we prove (Theorem 13) that even for a convex curve which is either polygonal or smooth, the number of points required may be arbitrarily large. Finally, in $\S 6$ we discuss whether any particular number of points will suffice for "most" curves.

The question answered by Theorem 1 originated in a context belonging to the interface between logic and geometry. We are grateful to S. Garfunkel for calling it to our attention and discussing it with the second named author; the logical ramifications will no doubt be explored elsewhere. We are also grateful to the Istituto Matematico of the Università di Perugia, Italy, whose hospitality during the summer of 1978 made our collaboration possible. Finally, we are very much indebted to the referee, whose detailed and creative critique of our original manuscript brought about very substantial improvements in the organization of the material, the depth and generality of our results, and the quality of our arguments. To $\mathrm{him} /$ her we owe the passage from plane curves to general continua in euclidean spaces and hence the present form of many of our results in $\S \S 2-4$.

2. Existence results. We first establish some notations and terminology. $K$ will denote a nonempty compact subset of some euclidean space $\boldsymbol{R}^{n}$ whose origin is denoted $\underline{0}$. Similarity and congruence are denoted by $\sim$ and $\cong$ respectively. $O(n)$ is the orthogonal group in euclidean $n$-space, consisting of the isometries which leave $\underline{0}$ fixed. If $G$ is a group of transformations, $K \widetilde{G}^{\prime} K^{\prime}$ means $K^{\prime}=T(K)$ for some $T \in G$. If $k \geqq 2$ is an integer, a minimal $k$ tuple in $K$ is a $k$-tuple $E \subset K$ such that if $E^{\prime} \subset K$ and $E^{\prime} \sim E$, it follows that $E^{\prime}$ is at least as large as $E$. We say that $K$ contains a minimal tuple if $K$ contains a minimal $k$-tuple for some integer $k \geqq 2$. There is a unique (degenerate, if $K$ consists of only one point) $(n-1)$-sphere of minimal radius which, together with its interior, contains $K$; this is the spanning sphere (or spanning circle if $n=2$ ) of $K$, denoted $S(K)$.

If $S$ is an $(n-1)$-sphere which together with its interior contains a compact set $K$, it is easy to verify the equivalence of the following three assertions: $S=S(K) ; S \cap K$ meets every closed hemisphere of $S$; and the center of $S$ is contained in the (automatically compact) convex hull of $S \cap K$. If the last assertion holds, then [4, p. 73, lemma] it holds with $S \cap K$ replaced by some subset of $S \cap K$ which contains at most $n+1$ points. Adjoining this subset of $S(K) \cap K$ to a minimal $k$-tuple yields (b) of the following proposition, while adding the endpoints of a diameter of $K$ to a minimal $k$-tuple yields (a). 
Proposition 2. If $K$ contains a minimal k-tuple, then:

(a) $K$ contains a subset $E_{1}$ of no more than $k+2$ elements such that $K \sim K^{\prime}$ and $E_{1} \subset K^{\prime}$ imply $K^{\prime} \cong K$.

(b) $K$ contains a subset $E_{2}$ of no more than $k+n+1$ elements such that $K^{\prime} \sim K$ and $E_{2} \subset K^{\prime}$ imply $S\left(K^{\prime}\right)=S(K)$.

This proposition applies in particular to the sets of Theorem 1.

Proposition 3. If $C$ is a convex plane curve, then $C$ contains a minimal 4-tuple, hence contains subsets $E_{1}$ and $E_{2}$, of no more than six and seven elements respectively, such that $K^{\prime} \sim K$ and $E_{1} \subset K^{\prime}$ imply $K^{\prime} \cong K$ while $K^{\prime} \sim K$ and $E_{2} \subset K^{\prime}$ imply $S\left(K^{\prime}\right)=S(K)$.

If $C$ is a convex or piecewise smooth simple closed plane curve all of whose corner angles are $>60^{\circ}$, the same assertions hold with 3-tuple, five and six in place of 4-tuple, six and seven respectively.

Proof. In the first instance note that $C$ has an inscribed parallelogram (indeed, a simple continuity argument shows that every point of $C$ is the vertex of an inscribed rhombus) and that the diameters of inscribed parallelograms are bounded below by area (C)/2 diameter (C); by a standard compactness argument one obtains a minimal inscribed copy of any inscribed parallelogram. A similar compactness argument produces a minimal equilateral triangle with vertices on $C$ in the second instance, since Proposition 10 below (which does not depend on Proposition 3) guarantees the existence of at least one equilateral triangle inscribed in $C$.

We have not ascertained whether $E_{1}$ can ever be strictly smaller than the smallest possible $E_{2}$, or whether the bounds six, seven, five, six are best possible.

THEOREM 4. Every compact set $K$ contains a finite subset $E$ such that $K^{\prime} \cong K$ and $E \subset K^{\prime}$ together imply $K^{\prime}=K$.

We thank the referee for this version of the theorem, as well as its proof. In view of Proposition 2, it immediately implies the following, which by Proposition 3 is a generalization of Theorem 1 .

THEOREM 5. Every compact set $K$ which contains a minimal tuple also contains a finite subset $E$ such that $K^{\prime} \sim K$ and $E \subset K^{\prime}$ together imply $K^{\prime}=K$.

Proof of Theorem 4. By taking (as in the proof of (b) of Proposition 2) no more than $n+1$ points from $K$ and assuming that 
$S(K)$ is centered at $\underline{0}$, we may reduce the proof of Theorem 4 to that of the following assertion:

(*) Every compact set $K$ contains a finite subset $E$ such that $K^{\prime} \underset{O(n)}{\widetilde{1}} K$ and $E \subset K^{\prime}$ imply $K^{\prime}=K$.

We shall prove $\left(^{*}\right)$ completely in dimension $n=2(n=1$ is trivial), and then indicate the argument for general $n$.

If $n=2$, let $G$ be the subgroup of $O(2)$ consisting of those transformations which leave $K$ fixed. Suppose the conclusion of $\left(^{*}\right.$ ) were false. Let $E_{1} \subset E_{2} \subset \cdots \subset E_{m} \subset \cdots$ be a sequence of finite subsets of $K$ such that $E_{m}$ is a $1 / m$-net of $K$. There is $T_{m} \in O(2)$ such that $T_{m}\left(E_{m}\right) \subset K$ but $T_{m}(K) \neq K$. Passing to a subsequence, we may suppose $T_{m} \rightarrow T$ in $O(2)$, and clearly $T \in G$. Replacing $E_{m}$ by $T\left(E_{m}\right)$ and $T_{m}$ by $T_{m} T^{-1}$, we may suppose $T_{m} \rightarrow I_{2}$ the identity transformation. Thus the $T_{m}$ are (for large $m$ ) rotations by angles $\theta_{m} \neq 0$ with $\theta_{m} \rightarrow 0$. Since $G \neq O(2), K$ must intersect some circle $S$ centered at $\underline{0}$ in a nonempty proper closed subset, which contains the endpoints $A$ and $B$ of an open arc $J$ in $S \backslash K$. For $m$ large, $T_{m}$ must take $A$ or $B$ into $J$. Hence if we replace each $E_{m}$ by $E_{m} \cup\{A, B\}$ and repeat the above reasoning, we obtain a contradiction.

The general case is handled with a similar argument and induction. Suppose $n>2$ and we know $\left(^{*}\right)$ in dimension $n-1$. Construct $G \subset O(n), E_{m}$ and $T_{m}$ as before (assuming (*) false for $K$ ). $K$ must intersect some sphere $S$ centered at $\underline{0}$ in a nonempty proper closed subset. Choose an open ball $B$ centered on $S$ and with maximal possible radius subject to $B \cap(S \cap K)=\varnothing$. If $S^{\prime}$ is the sphere which bounds $B, S \cap S^{\prime}$ is an $(n-2)$-dimensional sphere (or a point) and maximality of $B$ shows that (if $S \cap S^{\prime}$ is not a point) $K \cap\left(S \cap S^{\prime}\right)$ meets every closed hemisphere of $S \cap S^{\prime}$, so contains a finite subset $F$ with the same property; if $S \cap S^{\prime}=\{P\}$ a point take $F=\{P\}$. For $m$ large, $T_{m}$ must take at least one point of $F$ into $B \cap S$, unless $T_{m}$ leaves invariant the line through $\underline{0}$ and the center of $B$. Replacing $E_{m}$ by $E_{m} \cup F$ in our reasoning, we see that we may recoordinatize so that for large $m, T_{m}=I_{1} \times U_{m}$ with $I_{1}$ the identity transformation in the first variable and $U_{m} \in O(n-1)$ the orthogonal group on the last $n-1$ variables.

For each real number $t$ let $H_{t}$ be the subgroup of those $U \in$ $O(n-1)$ such that $I_{1} \times U$ leaves $K_{t}=K \cap\left(\{t\} \times \boldsymbol{R}^{n-1}\right)$ fixed, and let $H$ be the intersection of all the $H_{t}$ (that is, $H$ consists of those $U$ such that $I_{1} \times U$ leaves $K$ fixed). It follows from standard facts about Lie groups [e.g., Th. 50 of 3] that there are finitely many $t$, say $t_{1}, \cdots, t_{k}$, such that $H=H_{t_{1}} \cap \cdots \cap H_{t_{k}}$ and $K_{t_{j}} \neq \varnothing$. Using $\left(^{*}\right)$ for $n-1$ there are finite subsets $F_{j}$ of $K_{t_{j}}$ such that $K_{I_{1} \times O(n-1)} K_{t_{j}}$ 
and $F_{j} \subset K^{\prime}$ imply $K^{\prime}=K_{t_{j}}$. Now replace $E_{m}$ by $E_{m} \cup F_{1} \cup \cdots \cup F_{k}$ and repeat the reasoning. For $m$ large, $T_{m}=I_{1} \times U_{m}$ where $U_{m}\left(F_{j}\right) \subset$ $K_{t_{j}}$, hence $U_{m} \in H_{t_{j}}$ and so $U_{m} \in H$ or, what is the same, $T_{m} \in G$, a contradiction. This completes the proof of $\left(^{*}\right)$ and of Theorem 4.

3. The failure of existence. The following theorem describes what must occur for Theorem 5 to fail. It shows that Theorem 1 can be extended to many additional curves, for instance to piecewise smooth closed (but not necessarily simple) plane curves, and to analogous higher dimensional objects such as piecewise smooth closed surfaces. In addition, it should motivate the example which appears after it.

Roughly, the point $P$ whose existence is proved must be such that all of $K$ reproduces itself in $K$ within every neighborhood of $P$. Thus if $K$ is, say, a curve, it must have a highly singular behavior at $P$.

THEOREM 6. Suppose the compact set $K$ does not contain a finite set $E$ such that $K^{\prime} \sim K$ and $E \subset K^{\prime}$ imply $K^{\prime}=K$; equivalently, $K$ does not contain a minimal tuple. Then there are a point $P \in K$ and a sequence of similarities $\left(T_{m}\right)$ with the following property: for every neighborhood $N$ of $P, K \subset \lim _{m \rightarrow \infty} T_{u}(N \cap K)$ in the sense that for every $\varepsilon>0$ there is an $m_{0}$ such that for all $m>m_{0}$ every point of $K$ is within $\varepsilon$ of some point of $T_{m}(N \cap K)$.

Proof. Pick a sequence of finite subsets of $K, E_{1} \subset E_{2} \subset \cdots \subset$ $E_{m} \subset \cdots$, such that $E_{m}$ is a $1 / m$-net of $K$. $K$ contains no minimal copy of $E_{m}$, so there is a similarity $T_{m}$ such that $T_{m}^{-1}\left(E_{m}\right)$ is a subset of $K$ of diameter $<1 / m$. Passing to a subsequence, we may suppose that the sets $T_{m}^{-1}\left(E_{m}\right)$ converge to a point $P \in K$. This $P$ and these $T_{m}$ will do.

We shall now construct a simple closed curve $C$ which does not contain a minimal tuple. It will be clear that this curve can be rectifiable and, in addition, enjoy either of the following two mutually exclusive properties (except on one side of one point): polygonal (as in the particular example we exhibit) or infinitely differentiable (obtained by "rounding the corners" in our example). Precisely, $C$ can be obtained as the image of a continuous function $F:[0,1] \rightarrow \boldsymbol{R}^{2}$ which is one-to-one on $[0,1)$ and such that, for each $c \in(0,1)$, the restriction of $F$ to $[0, c]$ is either piecewise affine or infinitely differentiable with nowhere vanishing derivative.

EXAMPLE 7. We construct $C$ in stages $C_{1} \subset C_{2} \subset \cdots \subset C_{m} \subset \cdots$ 
with $C=\cup C_{m}$. Fix a positive number $b$. Each $C_{m}$ will be a compact polygonal arc which, except for the presence of the segment $J=$ $\{(x, 0): 0 \leqq x \leqq b\}$, will lie completely in $\left\{(x, y): x \geqq \delta_{m}, y>0\right\}$ where $\left(\delta_{m}\right)$ is a sequence of positive numbers tending to $0 . C_{m}$ will end at a point $P_{m}$ in the open first quadrant. No point of $C_{m}$ will lie directly to the left of $P_{m}$, and the points $P_{m}$ will converge to $\underline{0}$. Finally, all of $C_{m+1} \mid C_{m}$ will lie to the left of and no higher than $\bar{P}_{m}$, so will be at least as close to $\underline{0}$ as $P_{m}$, whence $\cup C_{m}$ will in fact be a simple closed curve $C$.

It is only necessary to describe the enlargement of $C_{m}$ to $C_{m+1}$. Starting at $P_{m}$, draw an arc going left, then down, then left, then up, staying in the open first quadrant, not meeting $C_{m}$, and ending as close as desired to $\underline{0}$. Using the endpoint of this "connecting link" as a starting point, construct a miniature copy of $C_{m}$ which meets neither the link nor $C_{m} \cdot C_{m+1}$ is the union of $C_{m}$, the link, and the miniature copy of $C_{m}$.

The sets $C \backslash C_{m}$ converge to $\underline{0}$. Any finite subset of $E$ is contained in some $C_{m}$ which is reproduced in each $C \backslash C_{m^{\prime}}$ for $m^{\prime} \geqq m$, so $C$ cannot contain a minimal tuple; clearly $\underline{0}$ is the point whose existence is specified by Theorem 6 .

A sample version of the first three stages with connecting link is presented in Figure 1 below.
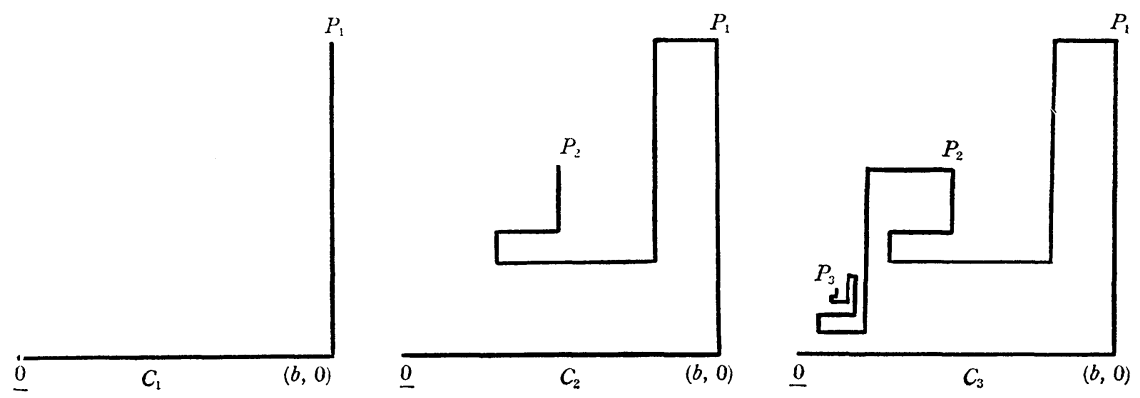

Figure 1

4. Particular sets in the plane. We define the (similarity) index $s(K)$ of the compact set $K$ to be the smallest possible cardinality of a subset $E$ of $K$ such that $K^{\prime} \sim K$ and $E \subset K^{\prime}$ imply $K^{\prime}=K$; the definition of oriented (similarity) index $o(K)$ is analogous, with replaced by $\tilde{f}$, which will denote orientation-preserving similarity. These indices sometimes coincide (e.g., regular polygon) and sometimes differ (e.g., nonisosceles triangle). Clearly $1 \leqq o(K) \leqq s(K) \leqq$ $\pm \infty$, and if $K$ contains at least three points then $o(K) \geqq 3$; further, if $o(K)$ is finite then so is $s(K)$. If $K$ and $K^{\prime}$ are exhibited as lists of points, say $\{P, Q, \cdots\}$ and $\left\{P^{\prime}, Q^{\prime}, \cdots\right\}$, the labelings are assumed 
to be so chosen that any similarity between $K$ and $K^{\prime}$ which we discuss makes points labelled with the same letter correspond. In this section we shall be concerned only with plane sets.

THEOREM 8. If $C$ is circle, $o(C)=s(C)=3$. If $K$ is a plane continuum which separates the plane and is not a circle, then $s(K) \geqq$ $o(K)>3$.

The hypothesis on separation is really necessary. If $K=\left\{\left(x, x^{2}\right)\right.$ : $-1 \leqq x \leqq 1\}$, then $K$ is not a subset of a circle, but $S(K)=3$ (take the three points $(-1,1),(0,0)$ and $(1,1))$. The proof of Theorem 8 is contained largely in Propositions 10 and 11, the proofs of which in turn require some topological preliminaries (which may be ignored $K$ is a simple closed curve).

Let $K$ be a plane continuum, $\hat{K}$ the union of $K$ and the bounded components of its complment, and $U$ a component of the interior of $\hat{K}$. If $\partial$ denotes topological boundary then $\partial U \subset \partial \hat{K} \subset \partial K \subset K$ and $[1, \S \mathrm{VI} .4] \partial U$ is connected.

\section{Lemma 9. If $A \in \partial U$ then $\partial U \backslash\{A\}$ is connected.}

Proof. If not $\partial U \backslash\{A\}=H_{1} \cup H_{2}$ where the $H_{j}$ are disjoint, nonempty, and open and closed in $\partial U \backslash\{A\}$. By [1, Th. IV. 10.1] their closures are $\bar{H}_{j}=H_{j} \cup\{A\}$. Let $\hat{H}_{j}$ be the union of $\bar{H}_{j}$ and those (necessarily bounded) components $V$ of the complement of $\partial U$ such that $\partial V \subset \bar{H}_{j}$. The $\hat{H}_{j}$ are compact, $\hat{H}_{1} \cap \hat{H}_{2}=\{A\}$, and $\hat{H}_{1} \cup \hat{H}_{2}$ has $U$ as a bounded component of its complement, so by [1, Cor. 1 to Th. V. 9.2] at least one of the $\hat{H}_{j}$, say $\hat{H}_{1}$, has disconnected complement.

Let $W$ be the unbounded component of the complement of $\bar{U} . W$ contains the complement of $\hat{K}$, so $H_{2} \subset \partial \hat{K} \subset \bar{W}$ whence $H_{2} \cup W$ is connected, and so is the union of $H_{2} \cup W$ with those bounded components $V$ of the complement of $\partial U$ such that $\partial V \cap H_{2} \neq \varnothing$; but this union is the complement of $\hat{H}_{1}$. This contradiction establishes the lemma.

The following result is due to M. L. O'Connor [2]; as her work has not yet been published, we offer here a proof incorporating the referee's improvements of our original argument for simple closed curves.

Proposition 10. If $K$ is a plane continuum which separates the plane and $E$ is any noncollinear triple of points, then $K$ contains infinitely many triples $E^{\prime} \widetilde{+} E$. 
Proof. Let $U$ be a component of the interior of $\hat{K}$. We shall show that the set of vertices of the $E^{\prime} \widetilde{+} E$ which are contained in $\partial U$ is dense in $\partial U$. Let $E=\{P, Q, R\}$ with longest side $\overline{Q R}$.

Let $A$ be any point of $U$, and let $P_{1}$ be a point nearest to $A$ on $\partial U$. The circle $S$ with center $A$ and radius $\overline{A P}_{1}$ has its interior in $U$. Choose $Q_{1}, R_{1}$ on $S \backslash\left\{P_{1}\right\}$ so that $\left\{P_{1}, Q_{1}, R_{1}\right\} \widetilde{f} E$; then $Q_{1}$ and $R_{1}$ lie on $\bar{U}$. Rotate $\left\{P_{1}, Q, R_{1}\right\}$ about $P_{1}$ until $Q_{1}$ or $R_{1}$ meets $\partial U$, say $Q_{1}$; one obtains $\left\{P_{1}, Q_{1}^{\prime}, R_{2}\right\} \widetilde{+} E$ with $Q^{\prime} \in \partial U$ and $R_{2} \in \bar{U}$, and $Q^{\prime}$ is close to any point to which $A$ is close.

If $P^{\prime} \in \partial U \backslash\left\{Q^{\prime}\right\}$, let $R\left(P^{\prime}\right)$ be that point such that $\left\{P^{\prime}, Q^{\prime}, R\left(P^{\prime}\right)\right\} \widetilde{+}$ $E$. Then $R(\cdot)$ is a continuous function on the connected (Lemma 9) set $\partial U \backslash\left\{Q^{\prime}\right\}, R\left(P_{1}\right)=R_{2} \in \bar{U}$, and, if $P_{2}$ is a point of $\partial U$ (equivalently, of $\bar{U})$ at maximal distance from $Q^{\prime}$ then $\overline{Q^{\prime} R\left(P_{2}\right)} \geqq \overline{Q^{\prime} P_{2}}$ implies that $R\left(P_{2}\right) \notin U$, hence for some $P^{\prime}, R^{\prime}=R\left(P^{\prime}\right) \in \partial U$.

Proposition 11. If $K$ is a plane continuum which separates the plane and is not a convex curve, and if $E$ is any collinear triple of points, then $K$ contains infinitely many triples $E^{\prime} \widetilde{+} E$.

Proof. Let $U$ be a component of the interior of $\hat{K}$, and let $E=$ $\{P, Q, R\}$ with $Q$ an interior point of the segment from $P$ to $R$.

Suppose $\bar{U}$ is not convex. Take $P_{1}, R_{1}$ on $\partial U$ such that the interior of the segment from $P_{1}$ to $R_{1}$ does not meet $\bar{U}$. Let $A \in U$ and let $R^{\prime}$ be a point of $\partial U$ closest to $A$. For $P^{\prime} \in \partial U \backslash\left\{R^{\prime}\right\}$ let $Q\left(P^{\prime}\right)$ be such that $\left\{P^{\prime}, Q\left(P^{\prime}\right), R^{\prime}\right\} \widetilde{+} E$. If $A$ is close to $R_{1}$ then $R^{\prime}$ is close to $R_{1}$ and $Q\left(P_{1}\right) \notin \bar{U}$. If $P_{2} \in \partial U \backslash\left\{R^{\prime}\right\}$ is such that the interior of the segment from $P_{2}$ to $R^{\prime}$ lies in $U$, then $Q\left(P_{2}\right) \in U$. Hence for some $P^{\prime}, Q^{\prime}=Q\left(P^{\prime}\right) \in \partial U$. There are infinitely many possible $R_{1}$, so the proof is complete in this case.

Suppose $\bar{U}$ is convex, so $\partial U$ is a convex curve $C$ which we may assume contains no segment. Let $A \in C$ be a point of accumulation of $K \backslash C$. Assume for definiteness that $\overline{Q R} \geqq \overline{P Q}$. Pick $R_{1} \in C \backslash\{A\}$.

If $A$ a point of accumulation of $K \cap U$, for fixed $Q^{\prime} \in K \cap U$ define $P(\cdot)$ from $C$ to itself so that $P\left(R^{\prime}\right), Q^{\prime}$ and $R^{\prime}$ are distinct and collinear. If $Q^{\prime}$ is close enough to $A$ then $\overline{Q^{\prime} R_{1} / P\left(R_{1}\right) Q^{\prime}} \geqq \overline{Q R \backslash P Q \text {, }}$ while if $R_{2}=P\left(R_{1}\right)$ then $P\left(R_{2}\right)=R_{1}$ and $\overline{Q^{\prime} R_{2} / P\left(R_{2}\right) Q^{\prime}} \leqq \overline{P Q / Q R} \leqq$ $\overline{Q R / P Q}$, so for some $R^{\prime} \in C, \overline{Q^{\prime} R^{\prime} / P\left(R^{\prime}\right) Q^{\prime}}=\overline{Q R / P Q}$, and taking $P^{\prime}=$ $P\left(R^{\prime}\right)$ gives $\left\{P^{\prime}, Q^{\prime}, R^{\prime}\right\} \widetilde{+} E$.

If $A$ is a point of accumulation of $K \backslash \bar{U}$, for fixed $P^{\prime} \in K \backslash \bar{U}$ define $Q(\cdot)$ from $C$ to the plane so that $\left\{P^{\prime}, Q\left(R^{\prime}\right), R^{\prime}\right) \tilde{+} E$. If $R_{2}$ is such that the line through $P^{\prime}$ and $R_{2}$ is a line of support for $C$, then $Q\left(R_{2}\right) \notin \bar{U}$; on the other hand, if $P^{\prime}$ is close enough to $A$ then $Q\left(R_{1}\right) \in$ 
$U$, hence for some $R^{\prime}, Q^{\prime}=Q\left(R^{\prime}\right) \in C$. The proposition is proved.

Proof of Theorem 8. If $C$ is a circle, there is nothing to prove. Let $K$ be as in the hypotheses, with spanning circle $S(K)$ assumed to be centered at $\underline{0}$. If $K$ is a union of circles centered at $\underline{0}$, then (being connected, but not a circle) it has nonempty interior, so clearly $s(K)=o(K)=+\infty$. Suppose $K$ is not a union of circles centered at $\underline{0}$. Then the similarities which leave $K$ invariant form a finite subgroup $G$ of $O(2)$. Thus it suffices to show that, for any triple $E \subset K, K$ contains infinitely many $E^{\prime} \tilde{+} E$.

If $E$ is not collinear, or $K$ is not a convex curve, this is immediate from Propositions 10 and 11. If $E$ is collinear and $K$ is a convex curve, then $K$ contains a segment, whence the assertion follows.

We now catalogue the indices of a few familiar simple closed plane curves $C$. We have $s(C)=o(C)=4$ if $C$ is a regular polygon, an isosceles triangle, a parallelogram, or an ellipse (not a circle). If $C$ is a nonisosceles triangle, $o(C)=4$ but $s(C)=5$; if $C$ is a trapezoid, $4 \leqq o(C) \leqq s(C) \leqq 5$. We indicate briefly how to prove these.

If $C$ is a regular polygon of $n>3$ sides (or a rectangle), take for $E$ the endpoints and an interior point of a (long, for a rectangle) side together with an interior point of the opposite side if $n$ is even (or for a rectangle) or the opposite vertex if $n$ is odd. For an ellipse take the endpoints of the two axes. For a rhombus which is not a square, take the vertices. For a trapezoid which is not a parallelogram take the vertices together with an interior point of the longer parallel side. For a parallelogram $C$ which is neither a rectangle nor a rhombus, take for $E$ the endpoints of the longer diagonal together with sufficiently nearby points of the longer sides, so chosen that $E$ itself consists of the vertices of a parallelogram.

The situation for triangles is rather more complicated. The arguments are based on the following simple lemma, together with a tedious enumeration of cases; we omit any details.

Lemma 12. Suppose $P, Q, R$ are the successive vertices of a nondegenerate triangle $T$ and $A, B, C, D$ are the successive vertices of a convex quadrilateral, both similarly oriented. Then there is $T^{\prime} \tilde{+}$ $T$ which contains $A, B, C$ and $D$ with $A$ and $B$ on the segment from $P^{\prime}$ to $Q^{\prime}$ if and only if (letting a letter stand for the magnitude of the angle at the corresponding vertex of a polygon):

$$
P \leqq A \leqq P+\pi-D \text { and } Q \leqq B \leqq Q+\pi-C .
$$

If in addition $A=P+\pi-D$ or $B=Q+\pi-C$, then there are 
uncountably many such $T^{\prime}$; otherwise there is only one.

5. Unboundedness of the index. While the results of the preceding section suggest that most familiar curves have small indices, it is nevertheless the case that there are "reasonable" curves with arbitrarily large indices. More is true: even to determine a curve among those obtained from it by rotation about a fixed center may require arbitrarily many points. Precisely:

THEOREM 13. Let $k$ be a positive integer. Then there are a positive integer $N(k)$ and a convex curve $C(k)$ (which may be taken polygonal or infinitely differentiable, as desired) such that, if $G(k)$ denotes the subgroup of $O(2)$ consisting of rotations about $\underline{0}$ through multiples of $2 \pi / N(k)$, then for any subset $E$ of $C(k)$ consisting of no more than $k$ points, there is $C^{\prime} \underset{G(k)}{\widetilde{C}} C(k)$ such that $C^{\prime} \neq C(k)$ but $E \subset C^{\prime}$.

The proof of this theorem rests on the following combinatorial fact.

Lemma 14. Let $k$ be a positive integer. Then there is a positive integer $n(k)$ such that if $n \geqq n(k)$ is an integer, then $\boldsymbol{Z}_{n}$, the (ring of) integers modulo $n$, can be partitioned into two subsets $A$ and $B$ such that the following two assertions hold:

(a) If $0 \neq m \in Z_{n}$ then $A \neq A+m \equiv\{x+m: x \in A\}$.

(b) If $K$ is any subset of $\boldsymbol{Z}_{n}$ containing no more than $k$ elements, then there is $m \in \boldsymbol{Z}_{n}, m \neq 0$, such that if $x \in K$ then $x \in A$ if and only if $x+m \in A$.

Before proving the lemma, we use it to prove the theorem.

Proof of Theorem 13. We shall construct $C=C(k)$ to be a polygon; it will be clear that by smoothing the corners one can obtain an infinitely differentiable $C$.

Let $N=N(k)$ be arbitrary subject to $N \geqq n(k), n(k)$ as in the lemma (so $N \geqq 2$ ), and let $A$ and $B$ be as in the lemma with $n=N$. If the plane has cartesian coordinates, for $j \in Z_{N}$ let $P_{j}=(\cos (2 j \pi / N)$, $\sin (2 j \pi / N))$. Choose $r$ so that $\cos (\pi / N)<r<1$ and for $j \in A$ let $Q_{j}=(r \cos ((2 j-1) \pi / N), r \sin ((2 j-1) \pi / N))$. Let $C$ be the convex polygon whose vertices are the $P_{j}$ for $j \in Z_{N}$ and the $Q_{j}$ for $j \in A$. For $j \in Z_{N}$ let $C_{j}$ denote the arc obtained by traversing $C$ counterclockwise from $P_{j-1}$ to $P_{j}$; thus $C_{j}$ is a closed segment if $j \in B$, and is the union of two non-collinear closed segments which meet at a common endpoint if $j \in A$. 
Now suppose $E$ is a subset of $C$ consisting of $k$ points $A_{1}, \cdots, A_{k}$ with $A_{i} \in C_{j(i)}, 1 \leqq i \leqq k$. Letting $K=\{j(i): 1 \leqq i \leqq k\}$, choose $m$ as in (b) of the lemma and let $C^{\prime}$ be the image of $C$ under rotation about $\underline{0}$ through an angle of $2 \pi m / N$. From (a) of the lemma $C^{\prime} \neq C$, and from (b) $E \subset C^{\prime}$.

Proof of Lemma 14. If $k$ and $n$ are integers with $n \geqq k \geqq 2$, by a $(k, n)$-configuration $F$ is meant a sequence of ordered pairs $\left\{\left(s_{i}, t_{i}\right)\right\}_{i=1}^{k}$ where $s_{i}=0$ or 1 and $\left\{t_{i}\right\}_{i=1}^{k}$ is a strictly increasing sequence of integers such that $t_{1}=0$ and $t_{k}<n$. A projection of $F$ is any of the (at most $n)$ sets $W=\left\{w+t_{i}\right\}_{i=1}^{k}$ where $w \in Z_{n}$. If $\{A, B\}$ is an ordered partition of $\boldsymbol{Z}_{n}$, by an appearance of $F$ in $\{A, B\}$ we mean a subset $K$ of $\boldsymbol{Z}_{n}$ consisting of $k$ elements which can be listed (perhaps in more than one way) as $w_{1}, \cdots, w_{k}$ in such a way that if $1 \leqq i \leqq k$ then $w_{i}=w_{1}+t_{i}$ while $s_{i}=0$ if and only if $w_{i} \in A$. Condition (b) of the lemma can now be restated as follows: if $F$ is any $(k, n)$-configuration which has an appearance in $\{A, B\}$, then $F$ has at least two distinct appearances in $\{A, B\}$. We shall prove the lemma with (b) replaced by the following stronger statement:

$\left(b^{\prime}\right)$ If $F$ is any $(k, n)$-configuration, then $F$ has at least two distinct appearances in $\{A, B\}$.

Fix an integer $k \geqq 2$. We need to know:

(c) There are a positive integer $n_{1}(k)$ and a positive number $\varepsilon=\varepsilon(k)$ such that if $n \geqq n_{1}(k)$ and $F$ is any $(k, n)$-configuration, then there are at least $n^{\varepsilon}$ pairwise disjoint projections of $F$.

Granted (c), suppose that $n \geqq \max \left\{n_{1}(k), n_{2}(k)\right\}$ where $n_{2}(k)$ is so large that $1+n^{\varepsilon} \log \left(1-2^{-k}\right) \leqq 0$ for $n \geqq n_{2}(k)$. If $F$ is a $(k, n)$ configuration which has $m\left(\geqq n^{\varepsilon}\right)$ pairwise disjoint projections then there are no more than

$$
m\left(2^{k}-1\right)^{m-1} 2^{k} 2^{n-k m} \leqq n^{\varepsilon}\left(2^{k}-1\right)^{n \varepsilon-1} 2^{k} 2^{n-k n^{\varepsilon}} \equiv \sigma
$$

ordered partitions of $Z_{n}$ in which $F$ appears at most once (the inequality follows from $\left.n \geqq n_{2}(k)\right)$. There are $\left(\begin{array}{l}n \\ k\end{array}\right) 2^{k}(k, n)$-configurations, so there are at most $\sigma\left(\begin{array}{l}n \\ k\end{array}\right) 2^{k}=2^{n} \tau$ ordered partitions of $Z_{n}$ in which $\left(b^{\prime}\right)$ fails, where

$$
\tau \equiv 2^{k}\left(\begin{array}{l}
n \\
k
\end{array}\right) n^{\varepsilon}\left(\frac{2^{k}-1}{2^{k}}\right)^{n^{\varepsilon}-1}
$$

tends to $O$ as $n$ gets large, so is $<1 / 2$ if $n \geqq n_{3}(k)$. In addition there are at most $\sum\left\{2^{d}: 1<d<n, d\right.$ divides $\left.n\right\}<(1 / 2) 2^{n}$ ordered partitions of $\boldsymbol{Z}_{n}$ for which (a) fails, so $n(k)=\max \left\{n_{1}(k), n_{2}(k), n_{3}(k)\right\}$ gives the lemma with (b) replaced by $\left(b^{\prime}\right)$. 
It remains to verify (c), which we shall do for any $\varepsilon(k)<1 / 2(k-1)$ and any $n_{1}(k) \geqq(k-1)^{2(k-1)}$ which is large enough so that

$$
n^{\varepsilon} \leqq\left[\frac{n^{1 / 2(k-1)}}{2(k-1)}\right] \text { for all } n \geqq n_{1}(k),
$$

where the brackets denote "integer part of". Indeed, let $n \geqq n_{1}(k)$ and let $F=\left\{\left(s_{i}, t_{i}\right)\right\}_{i=1}^{k}$ be any $(k, n)$-configuration and $W$ any of its projections. Let $u(i)=t_{i}-t_{i-1}$ if $2 \leqq i \leqq k$ and $u(1)=t_{1}-t_{k}+n$, and choose a permutation $\rho$ of $\{1, \cdots, k\}$ so that $u(\rho(1)) \leqq \cdots \leqq$ $u(\rho(k))$. Let $r=r(k, n)=n^{1 / 2(k-1)}$. There are two cases.

First, suppose $u(\rho(i+1)) \leqq r u(\rho(\mathrm{i}))$ for $1 \leqq i \leqq k-1$. Then for $1 \leqq i \leqq k$ we have $u(i) \geqq u(\rho(1)) \geqq r^{-(k-1)} u(\rho(k)) \geqq n^{-1 / 2}(n / k)=n^{1 / 2} / k$, so the $W_{j} \equiv W+j=\{w+j: w \in W\}$ for $0 \leqq j<n^{1 / 2} / k$ give at least $\left[n^{1 / 2} / k\right] \geqq n^{\varepsilon}$ pairwise disjoint projections of $F$.

Second, suppose there is an index $i, 1 \leqq i \leqq k-1$, for which $u(\rho(i+1))>r u(\rho(i))$. Then

$$
\mu \equiv u(\rho(1))+\cdots+u(\rho(i)) \leqq i u(\rho(i))<(k-1) u(\rho(i+1)) / r,
$$

so the $W_{j}^{\prime} \equiv W+(\mu+1) j$ for $0 \leqq j<u(\rho(i+1)) /(\mu+1)$ give a family of pairwise disjoint projections of $F$, of cardinality at least

$$
\left[\frac{u(\rho(i+1))}{\mu+1}\right] \geqq\left[\frac{r \mu}{(k-1)(\mu+1)}\right] \geqq\left[\frac{r}{2(k-1)}\right] \geqq n^{\varepsilon} .
$$

This proves (c), and so the lemma.

It is difficult to estimate $N(k)$ from the proof. For $k=2$, for instance, one can verify with $\varepsilon(2)=1 / 3$ that $n_{1}(2)=3^{6}$ and $n(2)=$ $129^{3}$ will work, and better estimates are hard to get.

However, if we represent $\boldsymbol{Z}_{16}$ as a sequence of places and put in each place a 0 or 1 according as the place belongs to $A$ or $B$, then it is easily verified that the string 0110100110010110 satisfies (a) and (b) (but not $\left(b^{\prime}\right)$ ) of the lemma, hence one can take $N(2)=16$.

6. Curves of small index. The values of the indices of particular curves given at the end of $\S 4$ suggest that "most" simple closed plane curves should have small index. Is there some index, or finite set of indices, which is "typical"? The group of orientation preserving similarity transformations is a four-parameter group, so four pieces of information "should" suffice to pinpoint one of its members. Thus we can hope that for "most" simple closed curves (or continua which separate the plane) $o(K)=4$ and $s(K)=4$ or 5 . We now present an interesting but inconclusive fact along these lines.

Let $W$ be the set of all simple closed plane curves, with the metric 


$$
\rho\left(C, C^{\prime}\right)=\sup _{P \in C} \inf _{Q \in C^{\prime}} d(P, Q)+\sup _{P \in C^{\prime}} \inf _{Q \in C} d(P, Q)
$$

where $d$ is the usual euclidean metric on the plane. Let $D$ be the subset of $W$ consisting of curves $C$ for which $s(C)=4$.

Proposition 15. $D$ is dense in $W$, and is the projection onto $W$ of $a G_{i}$-set in $W \times\left(\boldsymbol{R}^{2}\right)^{4}$.

The proposition remains true if we replace $W$ by any of various natural subsets, for instance, the set of convex curves. In particular, the set of convex curves with $\underline{0}$ in their interiors is complete under a metric equivalent to $\rho$, and the proposition is true for this set, even if we replace $\left(\boldsymbol{R}^{2}\right)^{4}$ by $S^{4}, S$ a circle.

Proof of Proposition 15. Consider a simple closed curve $C$ with the following properties: $\mathrm{C}$ contains a unique pair of points, say $A$ and $B$, a maximal distance apart; and exactly one member of this is an endpoint of a nondegenerate closed subarc $J$ of $C$ such that there is a circle $S$ of radius $r$ for which $C \cap S=J$, while if $S^{\prime}$ is any other circle of radius no more than $r$, then $C \cap S^{\prime}$ contains no more than two points. It is not hard to see that the set of such $C$-even those that are $C^{\infty}$-is dense in $W$, and we obtain $C \in D$ by taking for $E$ the set consisting of $A, B$ and two other points of $J$. Thus $D$ is dense in $W$.

Define $G_{n} \subset W \times\left(\boldsymbol{R}^{2}\right)^{4}(n=1,2, \cdots)$ as follows. Let $(C, \underline{\boldsymbol{P}}) \in W \times$ $\left(\boldsymbol{R}^{2}\right)^{4}$, so $\underline{\boldsymbol{P}}=\left(P_{1}, P_{2}, P_{3}, P_{4}\right)$ with $P_{j} \in \boldsymbol{R}^{2}$. We shall declare that $(C, \underline{P}) \in G_{n}$ provided that $C$ is not a circle, that the $P_{j}$ are distinct, and that there are positive numbers $\delta=\delta(C, \underline{P})$ and $\sigma=\sigma(C, \underline{P})$, $\delta<\sigma<1 / n$, satisfying:

$$
\begin{aligned}
& \operatorname{dist}\left(P_{j}, C\right) \equiv \inf _{Q \in C} d\left(Q, P_{j}\right)<1 / n, \\
& 3 \delta<\inf _{i \neq j} d\left(P_{i}, P_{j}\right) ;
\end{aligned}
$$

and whenever $\underline{Q}$ and $\underline{R}$ in $\left(\boldsymbol{R}^{2}\right)^{4}$ are such that $d\left(Q_{j}, P_{j}\right)<\delta$, $\operatorname{dist}\left(R_{j}, C\right)<$ $\delta, d\left(R_{i}, R_{j}\right) \geqq d\left(Q_{i}, Q_{j}\right) / n$, and $\left\{Q_{1}, Q_{2}, Q_{3}, Q_{4}\right\} \sim\left\{R_{1}, R_{2}, R_{3}, R_{4}\right\}$, then it follows that $d\left(R_{j}, P_{j}\right)<\sigma, 1 \leqq j \leqq 4$. It is not hard to check that $G_{n}$ is open, and that $(C, \underline{P})$ belongs to $\cap G_{n}$ precisely if $C \in D$ and $E=\left\{P_{1}, P_{2}, P_{3}, P_{4}\right\}$ works in the definition of $s(C) \leqq 4$. In other words, the projection of the $G_{\delta}$-set $\cap G_{n}$ onto $W$ is precisely $D$.

\section{REFERENCES}

1. M. H. A. Newman, Elements of the Topology of Plane Sets of Points, 2nd ed., Cambridge U. Press, 1954. 
2. M. L. O'connor, Some Metric Topological Invariants, U.C.L.A. Ph. D. Thesis, 1975.

3. L. Pontryagin, Topological Groups, Princeton U. Press, 1946.

4. W. Rudin, Functional Analysis, McGraw-Hill, 1973.

Received January 19, 1979 and in revised form July 19, 1979.

UNIVERSITE DE RENNÉS

38042 Rennes, France

AND

The University of Connecticut

StORRS, CT 06268

INSTITUT FOURIER

Laboratoire de Mathématiques Pures associé au C.N.R.S.

BP 116

38402 ST Martin D'heres, France 


\title{
PACIFIC JOURNAL OF MATHEMATICS
}

\section{EDITORS}

\author{
DoNALD BABBITT (Managing Editor) \\ University of California \\ Los Angeles, CA 90024 \\ Hugo RossI \\ University of Utah \\ Salt Lake City, UT 84112 \\ C. C. MOORE and ANDrew OGG \\ University of California \\ Berkeley, CA 94720
}

J. DugundJI

Department of Mathematics

University of Southern California

Los Angeles, CA 90007

R. FINN and J. MILGRAM

Stanford University

Stanford, CA 94305

\section{ASSOCIATE EDITORS}
R. Arens
E. F. BeCKenbaCH
B. H. NeumanN
F. WOLF
K. YoSHIDA

\section{SUPPORTING INSTITUTIONS}

UNIVERSITY OF ARIZONA

UNIVERSITY OF BRITISH COLUMBIA

CALIFORNIA INSTITUTE OF TECHNOLOGY

UNIVERSITY OF CALIFORNIA

MONTANA STATE UNIVERSITY

UNIVERSITY OF NEVADA, RENO

NEW MEXICO STATE UNIVERSITY

OREGON STATE UNIVERSITY

\author{
UNIVERSITY OF OREGON \\ UNIVERSITY OF SOUTHERN CALIFORNIA \\ STANFORD UNIVERSITY \\ UNIVERSITY OF HAWAII \\ UNIVERSITY OF TOKYO \\ UNIVERSITY OF UTAH \\ WASHINGTON STATE UNIVERSITY \\ UNIVERSITY OF WASHINGTON
}

The Supporting Institutions listed above contribute to the cost of publication of this Journal, but they are not owners or publishers and have no responsibility for its content or policies.

Mathematical papers intended for publication in the Pacific Journal of Mathematics should be in typed form or offset-reproduced, (not dittoed), double spaced with large margins. Please do not use built up fractions in the text of the manuscript. However, you may use them in the displayed equations. Underline Greek letters in red, German in green, and script in blue. The first paragraph or two must be capable of being used separately as a synopsis of the entire paper. Please propose a heading for the odd numbered pages of less than 35 characters. Manuscripts, in triplicate, may be sent to any one of the editors. Please classify according to the scheme of Math. Reviews, Index to Vol. 39. Supply name and address of author to whom proofs should be sent. All other communications should be addressed to the managing editor, or Elaine Barth, University of California, Los Angeles, California, 90024.

50 reprints to each author are provided free for each article, only if page charges have been substantially paid. Additional copies may be obtained at cost in multiples of 50 .

The Pacific Journal of Mathematics is issued monthly as of January 1966. Regular subscription rate: $\$ 102.00$ a year $(6$ Vols., 12 issues). Special rate: $\$ 51.00$ a year to individual members of supporting institutions.

Subscriptions, orders for numbers issued in the last three calendar years, and changes of address shoud be sent to Pacific Journal of Mathematics, P.O. Box 969, Carmel Valley, CA 93924, U.S.A Old back numbers obtainable from Kraus Periodicals Co., Route 100, Millwood, NY 10546.

\section{PUBLISHED BY PACIFIC JOURNAL OF MATHEMATICS, A NON-PROFIT CORPORATION}

Printed at Kokusai Bunken Insatsusha (International Academic Printing Co., Ltd.). 8-8, 3-chome, Takadanobaba, Shinjuku-ku, Tokyo 160, Japan. 


\section{Pacific Journal of Mathematics}

Vol. 93, No. $2 \quad$ April, 1981

Ilya Eugene Blum and Srinivasa Swaminathan, Continuous selections and

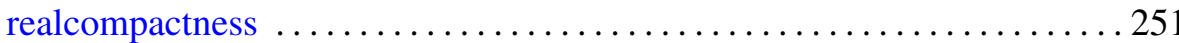

Lawrence James Brenton, Differentiably $k$-normal analytic spaces and extensions of holomorphic differential forms $\ldots \ldots \ldots \ldots \ldots \ldots \ldots 261$

Jo-Ann Deborah Cohen, Topologies on the ring of integers of a global field ................................................... 269

Robert Jay Daverman, Detecting the disjoint disks property $\ldots \ldots \ldots \ldots 277$

Edmund H. Feller, Rings where the annihilators of $\alpha$-critical modules are prime ideals ....................................... 299

Richard Elam Heisey and Henryk Torunczyk, On the topology of direct

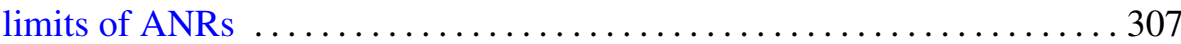

Gerald William Johnson and David Lee Skoug, Notes on the Feynman

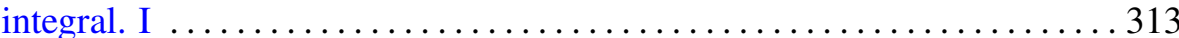

Michael S. Keane and Stuart Jay Sidney, Distinguishing a plane curve

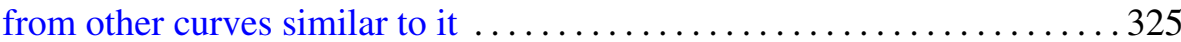

Leonid A. Luxemburg, On compact metric spaces with noncoinciding

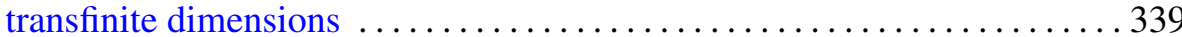

Chun Ming Ma, A uniqueness theorem for Navier-Stokes equations . . . . . . 387

Donald J. Newman and Theodore Joseph Rivlin, A characterization of the weights in a divided difference . . . . . . . . . . . . . . . . . . . 407

Marc Aristide Rieffel, $C^{*}$-algebras associated with irrational rotations $\ldots .415$

Kichi-Suke Saito, Invariant subspaces for finite maximal subdiagonal algebras

Frederic W. Shultz, Dual maps of Jordan homomorphisms and ${ }^{*}$-homomorphisms between $C^{*}$-algebras $\ldots \ldots \ldots \ldots$

Vsevolod Alekseevich Solonnikov, On the solvability of boundary and initial-boundary value problems for the Navier-Stokes system in domains with noncompact boundaries

Tavan Thomas Trent, New conditions for subnormality

L. E. Ward, Extending Whitney maps ..................

Leslie Wilson, Jets with regular zeros

Sergio Eduardo Zarantonello, The sheaf of $H^{p}$-functions in product 